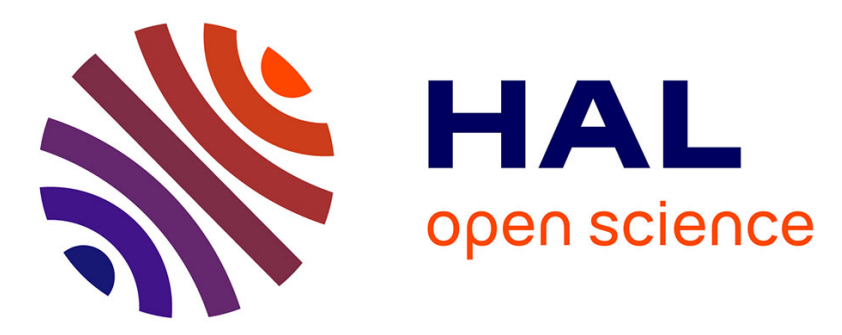

\title{
Multicenter Bonding in Ditetracyanoethylene Dianion: A Simple Aromatic Picture in Terms of Three-Electron Bonds
}

Benoît Braïda, Kevin Hendrickx, Dominik Domin, Joseph P. Dinnocenzo, Philippe C. Hiberty

\section{To cite this version:}

Benoît Braïda, Kevin Hendrickx, Dominik Domin, Joseph P. Dinnocenzo, Philippe C. Hiberty. Multicenter Bonding in Ditetracyanoethylene Dianion: A Simple Aromatic Picture in Terms of Three-Electron Bonds. Journal of Chemical Theory and Computation, 2013, 9 (5), pp.2276-2285. $10.1021 /$ ct $400290 \mathrm{n}$. hal-01627882

\section{HAL Id: hal-01627882 https://hal.science/hal-01627882}

Submitted on 9 Nov 2017

HAL is a multi-disciplinary open access archive for the deposit and dissemination of scientific research documents, whether they are published or not. The documents may come from teaching and research institutions in France or abroad, or from public or private research centers.
L'archive ouverte pluridisciplinaire HAL, est destinée au dépôt et à la diffusion de documents scientifiques de niveau recherche, publiés ou non, émanant des établissements d'enseignement et de recherche français ou étrangers, des laboratoires publics ou privés. 


\title{
Multicenter Bonding in Ditetracyanoethylene Dianion: A Simple Aromatic Picture in Terms of Three-Electron Bonds
}

\author{
Benoit Braida, ${ }^{*}{ }^{\dagger}$ Kevin Hendrickx, ${ }^{\dagger}$ Dominik Domin, ${ }^{\dagger, \|}$ Joseph P. Dinnocenzo, ${ }^{\ddagger}$ \\ and Philippe C. Hiberty*,§ \\ ${ }^{\dagger}$ UPMC Université Paris 06, CNRS UMR 7616, Laboratoire de Chimie Théorique, C. 137, 4 Place Jussieu, 75252 Paris Cedex 05, \\ France \\ ${ }^{\ddagger}$ University of Rochester, Department of Chemistry, Rochester, New York 14627, United States \\ ${ }^{\S}$ Laboratoire de Chimie Physique, UMR CNRS 8000, Groupe de Chimie Théorique, Université de Paris-Sud, 91405 Orsay Cédex, \\ France
}

\section{Supporting Information}

ABSTRACT: The nature of the multicenter, long bond in ditetracyanoethylene dianion complex $[\mathrm{TCNE}]_{2}^{2-}$ is elucidated using high level $a b$ initio Valence Bond (VB) theory coupled with Quantum Monte Carlo (QMC) methods. This dimer is the prototype of the general family of pancake-bonded dimers with large interplanar separations. Quantitative results obtained with a compact wave function in terms of only six VB structures match the reference $\operatorname{CCSD}(\mathrm{T})$ bonding energies. Analysis of the VB wave function shows that the weights of the VB structures are not
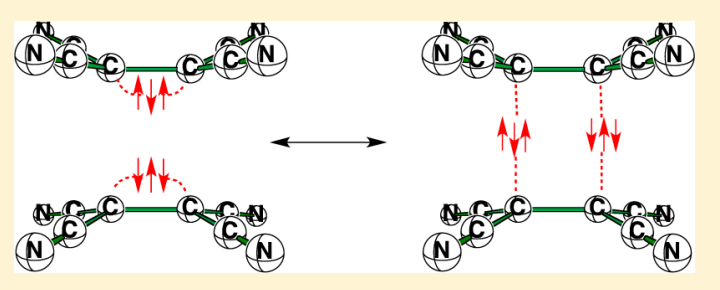
compatible with a covalent bond between the $\pi^{*}$ orbitals of the fragments. On the other hand, these weights are consistent with a simple picture in terms of two resonating bonding schemes, one displaying a pair of interfragment three-electron $\sigma$ bonds and the other displaying intrafragment three-electron $\pi$ bonds. This simple picture explains at once (1) the long interfragment bond length, which is independent of the countercations but typical of three-electron (3-e) CC $\sigma$ bonds, (2) the interfragment orbital overlaps which are very close to the theoretical optimal overlap of $1 / 6$ for a 3-e $\sigma$ bond, and (3) the unusual importance of dynamic correlation, which is precisely the main bonding component of 3-e bonds. Moreover, it is shown that the $[\mathrm{TCNE}]_{2}{ }^{2-}$ system is topologically equivalent to the square $\mathrm{C}_{4} \mathrm{H}_{4}{ }^{2-}$ dianion, a well-established aromatic system. To better understand the role of the cyano substituents, the unsubstituted diethylenic $\mathrm{Na}_{2}^{+}\left[\mathrm{C}_{2} \mathrm{H}_{4}\right]_{2}{ }^{2-}$ complex is studied and shown to be only metastable and topologically equivalent to a rectangular $\mathrm{C}_{4} \mathrm{H}_{4}{ }^{2-}$ dianion, devoid of aromaticity.

\section{INTRODUCTION}

Long, multicenter carbon-carbon bonding has been observed in dimers of a number of organic $\pi$-radicals. For example, the dimer of the tetracyanoethylene (TCNE) anion radical, referred to as $[\mathrm{TCNE}]_{2}{ }^{2-}$, was characterized as early as 1981 in the solid state. ${ }^{1}$ Since then other dianion dimers of the same type, i.e., exhibiting large interplanar separations, have been been reported, e.g., the dimers of tetracyanoquinodimethane $\left(\mathrm{TCNQ}^{-\bullet}\right){ }^{2}$ dicyanodichlorobenzoquinone $\left(\mathrm{DDQ}^{-\bullet}\right){ }^{3}$ chloranyl $\left(\mathrm{CA}^{-\bullet}\right)^{4}$ anion radicals, and others. ${ }^{4}$ As a prototype of such dimers, $[\mathrm{TCNE}]_{2}{ }^{2-}$ has been the object of many theoretical studies aimed at understanding the nature of the interactions that bind the two anion radicals. One outcome of these studies is a consensus that the bare $[\mathrm{TCNE}]_{2}{ }^{2-}$ dianion is unstable due to Coulomb and Pauli electronic repulsion, which needs be compensated by cation-anion attractive forces in the crystalline solid state, or by ion-solvent interactions in solution. $^{5-10}$ Thus, the cations or the solvent molecules act as the glue that keeps the two $[\mathrm{TCNE}]^{-\bullet}$ anions at a distance that enables the orbitals of each fragment to overlap. In the solid state, the most stable conformation has been shown to be the one in which the cations are in an equatorial position ( $I$ in
Scheme 1). ${ }^{6,11}$ Since the mere electrostatic interaction is attractive in this conformation, one may wonder if there exists a direct bond between the two anionic fragments, or if the dimer is only bound by electrostatics. Now the remarkable fact is that the distance $R$ separating the two anionic fragments is nearly independent of the size of the cation. ${ }^{6,12}$ Thus, among 14 different cation-mediated $[\mathrm{TCNE}]_{2}^{2-}$ crystals, the distance between the $[\mathrm{TCNE}]^{-\bullet}$ units was found to be $2.90 \pm 0.05 \AA$, whatever the nature of the cations, ranging from monatomic like $\mathrm{K}^{+}$or $\mathrm{Cs}^{+}$to bulky ones like $\left[\mathrm{Cr}\left(\mathrm{C}_{6} \mathrm{Me}_{3} \mathrm{H}_{3}\right)_{2}\right]^{+}$or $\left[i \operatorname{Pr}_{4} \mathrm{~N}\right]^{+}{ }^{6}$. Moreover, the same "magic" interfragment distance of $2.9 \AA$ was found in other dianions like $[\mathrm{TCNQ}]_{2}{ }^{2-}$ and $[\mathrm{CA}]_{2}{ }^{2-}$. A slightly longer distance $(3.05 \AA)$ was found for $[\mathrm{TCNE}]_{2}{ }^{2-}$ in solution. ${ }^{8}$ The near constancy of $R$ suggests that the interfragment distance is not controlled by the electrostatic cation-anion or solvent-anion interactions but by the direct interaction between the two anion radicals. Actually, the decisive proof of a direct interfragment bond in $[\mathrm{TCNE}]_{2}{ }^{2-}$ was provided by a calculation of Jung and Head-Gordon, ${ }^{7}$ where the 
Scheme 1. Schematic Equatorial (a) and Axial (b) Conformations for the $\mathrm{X}_{2}{ }^{+}[\mathrm{TCNE}]_{2}{ }^{2-}$ Complex
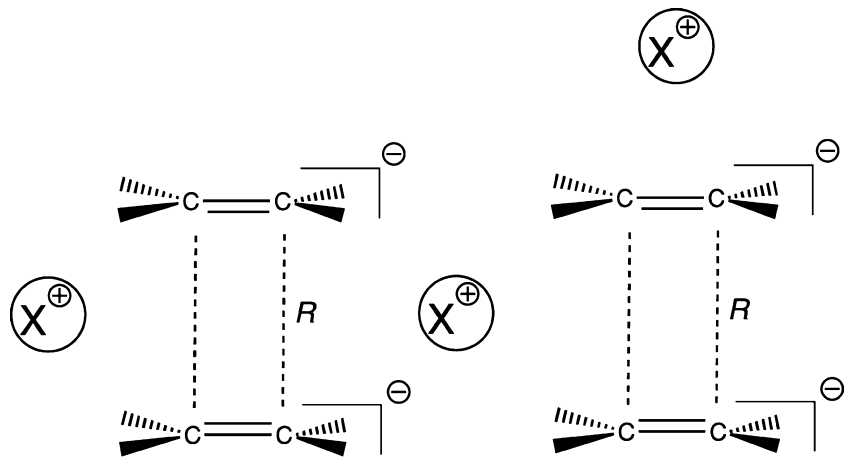

I

II

\section{equatorial cations axial cations $\mathrm{X}^{\oplus}$}

chosen geometry is one in which the $\mathrm{K}^{+}$countercations are in an axial position, respectively above and below the $[\mathrm{TCNE}]_{2}{ }^{2-}$ complex, like II in Scheme 1. This conformation is not the most stable geometry, but it is a particularly interesting one as the two $\mathrm{K}^{+}[\mathrm{TCNE}]^{-\bullet}$ fragments form two dipoles that point in opposite directions, so that the electrostatic component of the interaction must be repulsive. Thus, in this conformation, any attractive interaction between the two fragments must be entirely due to a direct bond between the two $[\mathrm{TCNE}]^{-\bullet}$ units. This is actually the case in the $\mathrm{K}_{2}^{+}[\mathrm{TCNE}]_{2}^{2-}$ axial complex, which is found to be more stable by $11.2 \mathrm{kcal} / \mathrm{mol}$ at an equilibrium distance than at infinite distance, at the MCQDPT/CASSCF $(2,2)$ level with counterpoise correction. ${ }^{7}$ Moreover, another sign of a direct interaction between the two TCNE fragments is the presence of a $(3,-1)$ bond critical point in the middle of each $\mathrm{C}-\mathrm{C}$ interlinkage, ${ }^{13}$ in accord with Bader's definition of a bond. ${ }^{14}$ Thus, it is definitely clear that there is a chemical bond between the two $[\mathrm{TCNE}]^{-\bullet}$ units, and that this bond contributes to the stability of the dimer. The next question is, of course, "What kind of bond is it?"

In the molecular orbital (MO) framework, each $[\mathrm{TCNE}]^{-\bullet}$ radical anion has an unpaired electron in a $\pi^{*}$ orbital. When the two $[\mathrm{TCNE}]^{-\bullet}$ fragments are facing each other in two parallel planes, the two $\pi^{*}$ orbitals overlap and combine to form a bonding $\mathrm{MO}$ of $\mathrm{b}_{2 \mathrm{u}}$ symmetry, as shown in Scheme 2. In accord, the interaction between the $[\mathrm{TCNE}]^{-\bullet}$ fragments has first been considered as a simple, albeit multicenter, stretched two-electron covalent $\mathrm{C}-\mathrm{C}$ bond, more precisely a cationmediated or solvent-mediated covalent bond. ${ }^{5,6}$ As the bond involves four carbon atoms, it was designated as a two-electron/ four-center bond, while different multicenter bonds in other dimers were identified as $n$-electron/m-center bond $\left(n \mathrm{e}^{-} / m \mathrm{c}\right)$, $n$ being the number of unpaired electrons in the fragments, e.g., $2 \mathrm{e}^{-/ 12 \mathrm{c}}$ for the phenalenyl dimer, ${ }^{10} 2 \mathrm{e}^{-} / 8 \mathrm{c}$ for the tetracyanopyrazine dimer, ${ }^{15}$ and so on. These designations, while topologically clear, do not provide much insight into the nature of the bonding interactions. Are these new types of bonds, or can the bonding be described in terms of known bonding types, e.g. two-electron bonds, dispersion interactions, or other elementary bonding types? The $2 \mathrm{e}^{-} / \mathrm{mc}$ designation is also potentially unsuitable because it implies that only two electrons participate in the interaction-a questionable assumption, as will be shown below.

Back to the $[\mathrm{TCNE}]_{2}{ }^{2-}$ complex, it has been noted that some features of the interfragment bonds are not fully compatible with a classical covalent nature. For example, it has been noted ${ }^{16}$ that electron density analysis shows electron depletion in the bonding region as reflected by the positive or close to zero Laplacian at the bond critical points, at variance with the negative Laplacians that are normally observed for covalent bonds. Besides, the average bond length of $2.9 \AA$ is intriguing. It is much longer than a standard $\mathrm{C}-\mathrm{C}$ two-electron bond $(1.54 \AA)$ and shorter than the sum of carbon van der Waals radii $(3.4 \AA)$. Moreover, Head-Gordon ${ }^{7}$ showed that the axial conformation is found unstable at the level of twoconfiguration calculation of the MCSCF type, i.e., at the $\operatorname{CASSCF}(2,2)$ level, while such a computational level normally describes very well a classical covalent bond like, e.g., the $\mathrm{C}-\mathrm{C}$ bond of ethane. This author also showed that adding a secondorder perturbative correction to the $\operatorname{CASSCF}(2,2)$ wave function, leading to the MCQDPT/CASSCF $(2,2)$ level, stabilizes the relative interaction energy of the two $\mathrm{K}^{+}[\mathrm{TCNE}]^{-\bullet}$ fragments by $\sim 23 \mathrm{kcal} / \mathrm{mol}$, which is much more than expected for a stretched $\mathrm{C}-\mathrm{C}$ covalent bond.

Scheme 2. Molecular Orbitals Arising from the Combinations of the $\pi$ and $\pi^{*}$ Orbitals of the $\left[\mathrm{TCNE}^{-}\right.$Fragments

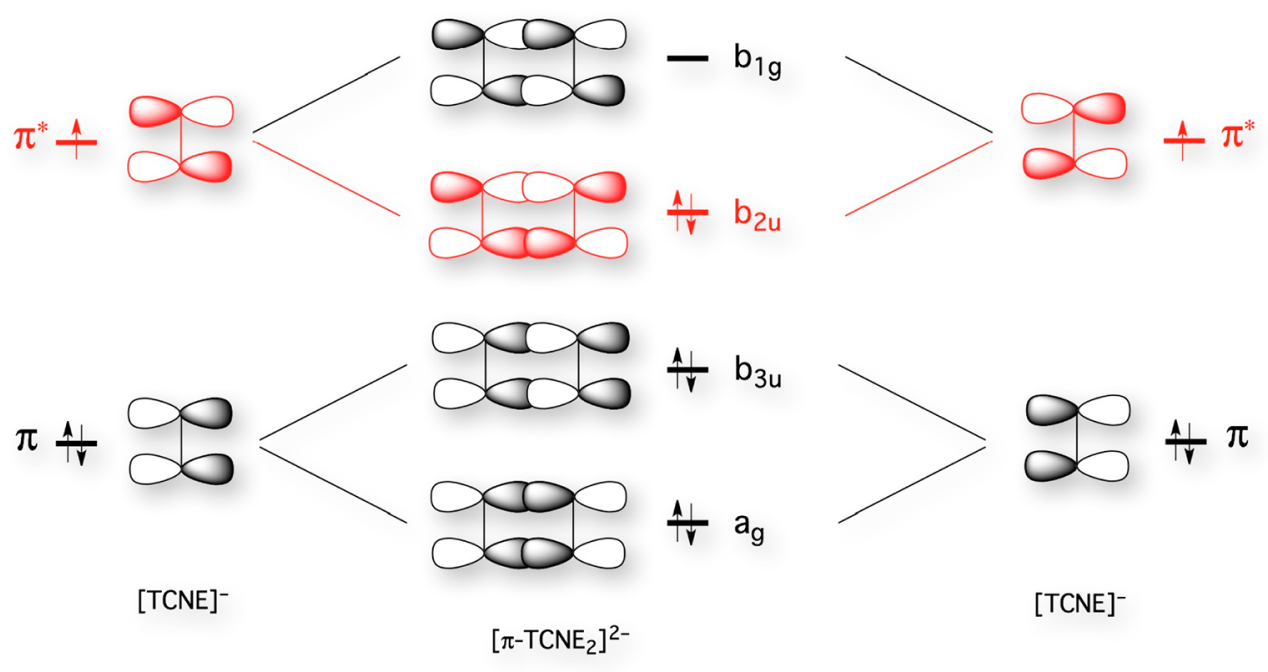


Calculations of a similar type with different geometries were subsequently performed by Novoa et al. ${ }^{9}$ Finally, the importance of dynamic correlation, and the irrelevance of a classical covalent scheme, was further emphasized in a recent calculation using the generalized valence bond method with subsequent configuration interaction (CI) by Tian and Kertesz, who suggested that bonding of the charge-shift type ${ }^{16}$ significantly contributes to the chemical bonding in $[\mathrm{TCNE}]_{2}{ }^{2-}$ complexes. $^{17}$

Jung and Head-Gordon ${ }^{7}$ and Novoa et al. ${ }^{11}$ addressed the question of the nature of the interfragment bond as follows. Having showed that taking dynamic correlation into account is critical in describing TCNE anion dimerization, they interpreted this finding as a sign of an important contribution of dispersion attractions between the two cofacial monomers, together with some interdimer $\pi$-bonding interactions. Now it should be noted that dynamic correlation may also be important in some bonding interactions that do not involve dispersion, like for example odd-electron bonding ${ }^{18}$ or chargeshift bonding. ${ }^{16 a}$ It follows that the quantitative contribution of dispersion, per se, is difficult to quantify, and that the exceptional magnitude of dynamical correlation is perhaps not to be entirely ascribed to dispersion, but possibly to some intradimer $\pi$-bonding interaction different from the twoelectron covalent type. Thus, it appears that the origin of the long four-center bond in $[\mathrm{TCNE}]^{-}$dimers and related compounds is still not a definitely settled matter. In the discussion below, we explore other possible types of bonding for $[\mathrm{TCNE}]_{2}{ }^{2-}$.

For a system displaying no attractive electrostatic forces, chemical bonds can be of the one-electron, two-electron, or three-electron type, if one leaves dispersion forces aside. ${ }^{19,20}$ At long distances, each TCNE unit has three electrons in its $\pi$ system and consequently displays a three-electron (3-e) $\pi$ bond (see Scheme 3 below). When the fragments are brought

Scheme 3. Representations of the Three-Electron $\pi$ Bond in the Ethylene Anion: (a) the Valence Bond Framework and (b) the Molecular Orbital Framework

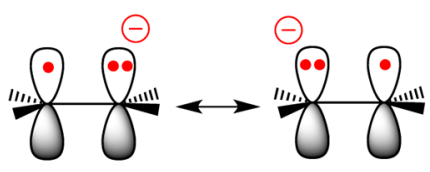

(a)

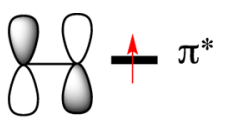

(b)

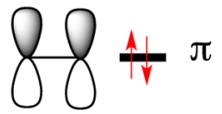

together, these intrafragment 3-e bonds can convert into a pair of interfragment 3-e bonds, as will be detailed below. Could such bonds, which have not been considered in previous theoretical studies, provide a way to understand the bonding in the anion radical dimers, and in particular the intriguing average bond length of $2.9 \AA$ ? Qualitatively speaking, there are a priori two arguments in favor of this type of bonding. First, calculated 3-e bonding energies are extremely sensitive to dynamic correlation. For example, the calculated bonding energy of $\mathrm{F}_{2}^{-}$, a 3-e bonded molecule, is close to zero at the HartreeFock level but increases to $\sim 30 \mathrm{kcal} / \mathrm{mol}$ when dynamic correlation is taken into account. ${ }^{18}$ Therefore, the same argument that was used for $[\mathrm{TCNE}]_{2}{ }^{2-}$ in favor of dispersion forces, ${ }^{7,11}$ based on the importance of dynamic correlation, could also be used to support the 3-e bonding model. Second, 3-e bonds are long bonds. Strikingly enough, an MP2 optimization of the 3-e $\mathrm{C}-\mathrm{C}$ bond length in the ethane anion radical, $\left[\mathrm{H}_{3} \mathrm{C}: \mathrm{CH}_{3}\right]^{-}$, gives an equilibrium distance of $2.65 \AA$, which is almost exactly the interfragment distance found in $[\mathrm{TCNE}]_{2}{ }^{2-}$ at a comparable level of calculation using the same basis set. ${ }^{7}$

Based on the reasons cited above, we were motivated to perform a theoretical study of $[\mathrm{TCNE}]_{2}{ }^{2-}$ using ab initio valence bond $(\mathrm{VB})$ theory to investigate the nature of the multicenter bond with the aim of providing insight that standard MO methods cannot give. The choice of a VB rather than an MO-based computational method is indeed ideal for our purpose for three reasons: (i) VB provides an extremely compact wave function made of a small number of VB structures which are expressed in the chemist's language and clearly interpretable in terms of bonding interactions, (ii) VB theory provides in particular a clearly identifiable resonating description of three-electron bonding interactions as we will see later on, and (iii) dynamic correlation can be included in the VB method without altering the compactness and interpretability of the wave function. Thus, the interpretation of the electronic structure in terms of a bonding model is made by directly analyzing the accurate wave function, and not by analyzing a lower-level one as is often the case. This latter feature is obviously quite critical owing to the importance of dynamic correlation in the problem at hand.

\section{COMPUTATIONAL METHODS}

A many-electron system wave function $\Psi$ in $\mathrm{VB}$ theory is expressed as a linear combination of Heitler-London-SlaterPauling (HLSP) functions, $\Phi_{K}$ in eq $1:{ }^{21}$

$$
\Psi=\sum_{K} C_{K} \Phi_{K}
$$

where $\Phi_{K}$ corresponds to "classical" VB structures and $C_{K}$ represents structural coefficients. The weights of the VB structures are defined by the Coulson-Chirgwin formula, ${ }^{22}$ eq 2 , which is the equivalent of a Mulliken population analysis in VB theory.

$$
W_{K}=C_{K}^{2}+\sum_{L \neq K} C_{K} C_{L}\left\langle\Phi_{K} \mid \Phi_{L}\right\rangle
$$

where $\left\langle\Phi_{K} \mid \Phi_{L}\right\rangle$ is the overlap integral of two VB structures.

There are several computational approaches for VB theory at the $a b$ initio level. ${ }^{23}$ In the VBSCF procedure, ${ }^{24}$ both the VB orbitals and structural coefficients are optimized simultaneously to minimize the total energy. As such, the VBSCF method takes care of the static electron correlation, but it lacks dynamic correlation. Among the electrons and orbitals, one distinguishes an active space, here the $\pi$ orbitals of each fragments and their electrons, from an inactive space (all the rest). The active space is treated at the VB level, and its electrons are explicitly correlated, while the inactive part of the molecule is described as a set of doubly occupied orbitals, so that the correlation of inactive electrons and the active-inactive correlation are not explicitly taken into account. An important feature of our VB calculations is that all the active orbitals are strictly localized on a single atom, like in the classical VB method, so as to ensure a clear correspondence between the mathematical expressions of the VB structures and their physical meaning, ionic or covalent.

Very recently, a mixed Valence Bond/Quantum Monte Carlo (VB/QMC) method was proposed which managed to provide 
high accuracy while keeping the full interpretative capabilities of classical valence bond methods. ${ }^{25}$ Basically, the VB/QMC wave function we utilized consists of a VBSCF determinant part multiplied by a Jastrow function that is included to account for electronic correlation. In this study, the Jastrow parameters, VB structure coefficients, and active orbitals are optimized simultaneously using the Variational Monte Carlo (VMC) optimization algorithm, while the inactive orbitals are taken from a previous VBSCF calculation and held fixed. This level is referred to here as the VB-VMC method. The VB-VMC wave function is projected onto the ground state using the Diffusion Monte Carlo (DMC) algorithm under the fixed-node approximation, a procedure that recovers most of the remaining correlation effects that are missing at the VB-VMC level and usually provides very accurate energy differences. ${ }^{25,26}$ This method is referred to here as VB-DMC. For both types of VB/ QMC calculations, the systematically convergent basis sets of Burkatzi et al. ${ }^{27,28}$ were used together with their corresponding pseudopotentials for $\mathrm{C}$ and $\mathrm{N}$. For $\mathrm{Na}$, only the pseudopotential is used in the calculation in order to ensure that true $\mathrm{Na}^{+}$ ions were considered. For the ditetracyanoethylene dianion $[\mathrm{TCNE}]_{2}{ }^{2-}$, in order to keep a reasonable computational cost, the original double- $\zeta$ polarized basis set was used for the four carbon atoms implied in the pancake bonding, whereas the polarization functions on the cyano substituents were removed from the calculation. The VBSCF part of the calculations was carried out with the Xiamen Valence Bond (XMVB) program, ${ }^{29,30}$ while the QMC calculations were carried out using the CHAMP program. ${ }^{31}$

The reliability of the $\operatorname{CCSD}(\mathrm{T})$ calculations, performed to get a reference dissociation energy curve for the $\mathrm{Na}_{2}{ }^{+}[\mathrm{TCNE}]_{2}{ }^{2-}$ complex, was ensured by means of the standard "T1 diagnostic," 32 which amounts to 0.015. A coupled-cluster calculation displaying a $\mathrm{T} 1$ value smaller than 0.02 is generally considered as reliable. ${ }^{33}$

The VB Structure Set and Its Relationship to MO Configurations. The active VB space, in the sense defined above, is made of the $\pi$ orbitals $\chi_{1}-\chi_{4}$ of both fragments and of the six electrons that occupy these orbitals. All the VB structures corresponding to this active space are generated. For such an active space of six electrons in four orbitals there are, in principle, $10 \mathrm{VB}$ structures, which are represented in Scheme 4 as 1-10 and correspond to the VB functions $\phi_{1}-\phi_{10}$. The most important ones are obviously $1-6$, which all have two singly occupied atomic orbitals (AO). The four remaining structures 7-10, represented by the smaller depictions in Scheme 4, all have one empty AO and three doubly occupied ones and bear four formal charges. As these latter VB structures are practically negligible at bonding distances, as will be shown below, they will not be considered in the qualitative interpretation of the VB wave functions.

As is well-known, any configuration state function in the MO framework can be mapped to VB structures, a transformation that can easily be done by hand in the six-electron/four-orbital case. ${ }^{19,34}$ Particularly interesting is the MO-VB correspondence for the Hartree-Fock configuration (eq 3) and first doubly excited configuration (eq 4), which constitute the two configurations involved in a GVB calculation.

$$
\left(\mathrm{a}_{\mathrm{g}}\right)^{2}\left(\mathrm{~b}_{3 \mathrm{u}}\right)^{2}\left(\mathrm{~b}_{2 \mathrm{u}}\right)^{2}=\mathcal{N}\left(+\phi_{1}+\phi_{2}-\phi_{3}-\phi_{4}-\phi_{5}-\phi_{6}\right)
$$

Scheme 4. The Set of 10 VB Structures That Are Necessary and Sufficient to Represent the $[\mathrm{TCNE}]_{2}{ }^{2-}$ Complex

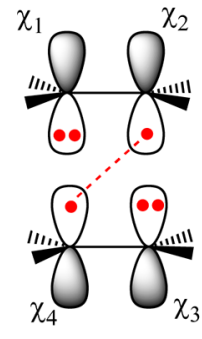

1
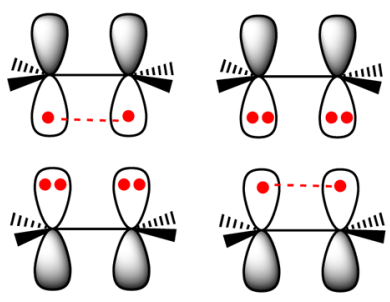

5

2
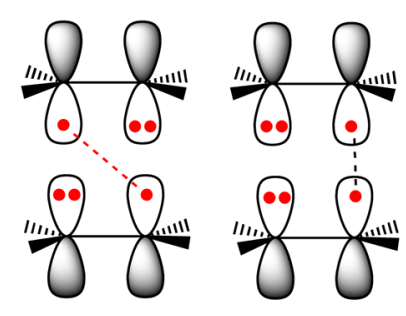

3
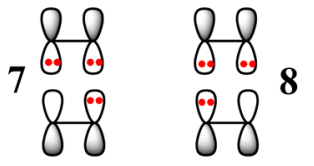

9

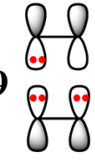

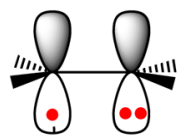

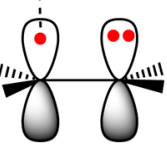

4

$$
\left(\mathrm{a}_{\mathrm{g}}\right)^{2}\left(\mathrm{~b}_{3 \mathrm{u}}\right)^{2}\left(\mathrm{~b}_{1 \mathrm{~g}}\right)^{2}=\mathcal{N}\left(-\phi_{1}-\phi_{2}+\phi_{3}+\phi_{4}-\phi_{5}-\phi_{6}\right)
$$

where $\mathcal{N}$ is a generic term standing for a normalization constant, and structures $\phi_{7}-\phi_{10}$ are ignored for the reasons stated above. It is noteworthy that mixing these two configurations necessarily yields a state in which the four VB structures 1-4 have equal coefficients. On the other hand, the coefficients of structures 5 and $\mathbf{6}$, which represent chargetransfer terms displaying a neutral TCNE fragment in front of a di-ionic one, are different from those of 1-4 owing to the configuration mixing. Thus, the covalent interaction that is inherent to a GVB or $\operatorname{CASSCF}(2,2)$ wave function would correspond to a VB wave function having identic weights for each of the VB structures 1-4, and a presumably very small amount of structures 5 and $\mathbf{6}$, since long covalent bonds are practically pure diradicals involving very few charge-transfer terms. Recall that GVB or CASSCF $(2,2)$ levels completely lack dynamic correlation and dispersion terms.

\section{RESULTS}

The geometries of the $\mathrm{Na}_{2}{ }^{+}[\mathrm{TCNE}]_{2}{ }^{2-}$ system have been fully optimized at the MP2 level, for a series of fixed interfragment distances using the same double- $\zeta$ basis set as for the VB/QMC calculations. Using these geometries, the equilibrium interfragment distance was subsequently reoptimized at the $\operatorname{CCSD}(\mathrm{T})$ level using the counterpoise corrections for the basis set superposition error (BSSE), a level referred to here as CP$\operatorname{CCSD}(\mathrm{T})$. This was done by plotting the dissociation curve, which was obtained by a series of single point energy calculations at the CP-CCSD $(\mathrm{T})$ level, using the cc-pVTZ basis set for the four central carbons and the lighter 6-31G(d) basis set on the cyano groups. A real minimum was found at an interfragment distance of $2.56 \AA$. The geometry of this equilibrium structure, displayed in Figure 1, is quite similar to that determined by Jung and Head-Gordon, ${ }^{7}$ with the cyano substituents slightly bending away from the center of the dimer 

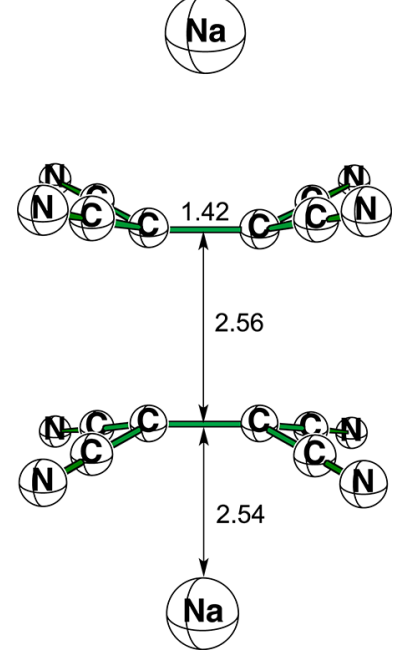

Figure 1. Optimized geometries of the $\mathrm{Na}_{2}{ }^{+}[\mathrm{TCNE}]_{2}{ }^{2-}$ system in its axial conformation.

as in the X-ray structure. ${ }^{4}$ As the fragments are pulled away from each other, the energy smoothly rises in a barrierless dissociation up to the separate fragments, lying $11.6 \mathrm{kcal} / \mathrm{mol}$ above the equilibrium structure at the $\operatorname{CP}-\operatorname{CCSD}(\mathrm{T})$ level (Table 1, entry 1). Once again, this result is in excellent

Table 1. Relative Energies, in $\mathrm{kcal} / \mathrm{mol}$, of the Equilibrium Structure of the $[\mathrm{TCNE}]_{2}^{2-}$ System, Relative to Separate Fragments

\begin{tabular}{lr}
{$[\mathrm{TCNE}]_{2}{ }^{2-}$} & $E\left(R_{\mathrm{eq}}\right)$ \\
$\operatorname{CCSD}(\mathrm{T})$ & -11.6 \\
$\operatorname{VB}-\mathrm{VMC}(\mathbf{1 - 4})$ & +5.4 \\
$\operatorname{VB}-\mathrm{VMC}(\mathbf{1 - 6})$ & -3.5 \\
$\operatorname{VB}-\mathrm{VMC}(\mathbf{1 - 1 0})$ & -5.2 \\
$\mathrm{VB}-\mathrm{DMC}(\mathbf{1 - 1 0})$ & -9.7 \\
\hline
\end{tabular}

agreement with the dissociation energy of $11.2 \mathrm{kcal} / \mathrm{mol}$ obtained by Jung Head-Gordon at the MCQDPT/CASSCF$(2,2)$ level with counterpoise correction. ${ }^{7}$ Of course, these values are lower limits for the true interaction energy since the electrostatic component is repulsive in this axial conformation.

The VB calculations of bonding energies for the $\mathrm{Na}_{2}{ }^{+}[\mathrm{TCNE}]_{2}{ }^{2-}$ complex are displayed in Table 1 , for both the ground state and various diabatic states. All VB calculations use the same geometries as the ones used above in the CP$\operatorname{CCSD}(\mathrm{T})$ calculations, for the complex and dissociated fragments. At the most accurate VB level, VB-DMC involving the whole set of structures 1-10 displayed in Scheme 4, the bonding energy amounts to $9.7(9) \mathrm{kcal} / \mathrm{mol}$ (Table 1, entry 5), thus very close to the $\mathrm{CP}-\mathrm{CCSD}(\mathrm{T})$ reference, showing that a compact 10-structure $\mathrm{VB}$ wave function is able to capture the essential physics of the TCNE-TCNE interaction, provided dynamic correlation is properly included. The VB-VMC level, which includes all static correlation and a large part but not all of the dynamic correlation effects, ${ }^{25,26}$ still provides a bonding energy of $5.2(9) \mathrm{kcal} / \mathrm{mol}$ (Table 1, entry 4). Albeit less accurate than VB-DMC, the VB-VMC level has the advantage of being appropriate for the calculation of diabatic states, involving a restricted set of VB structures (see, e.g., entries 2 and 3 in Table 1). This added flexibility makes the VB-VMC level a useful tool for interpretative purposes and will be used in what follows for deeper analyses of the VB results.

The four active orbitals $\chi_{1}-\chi_{4}$, as they arise from the orbital optimization at the VB-VMC level, are schematically represented in Scheme 5, whereas the exact contour plot of

Scheme 5. The Four Fragment Orbitals Involved in the VB Calculations of $\mathrm{Na}_{2}{ }^{+}[\mathrm{TCNE}]_{2}{ }^{2-}$ and Their Mutual Overlaps
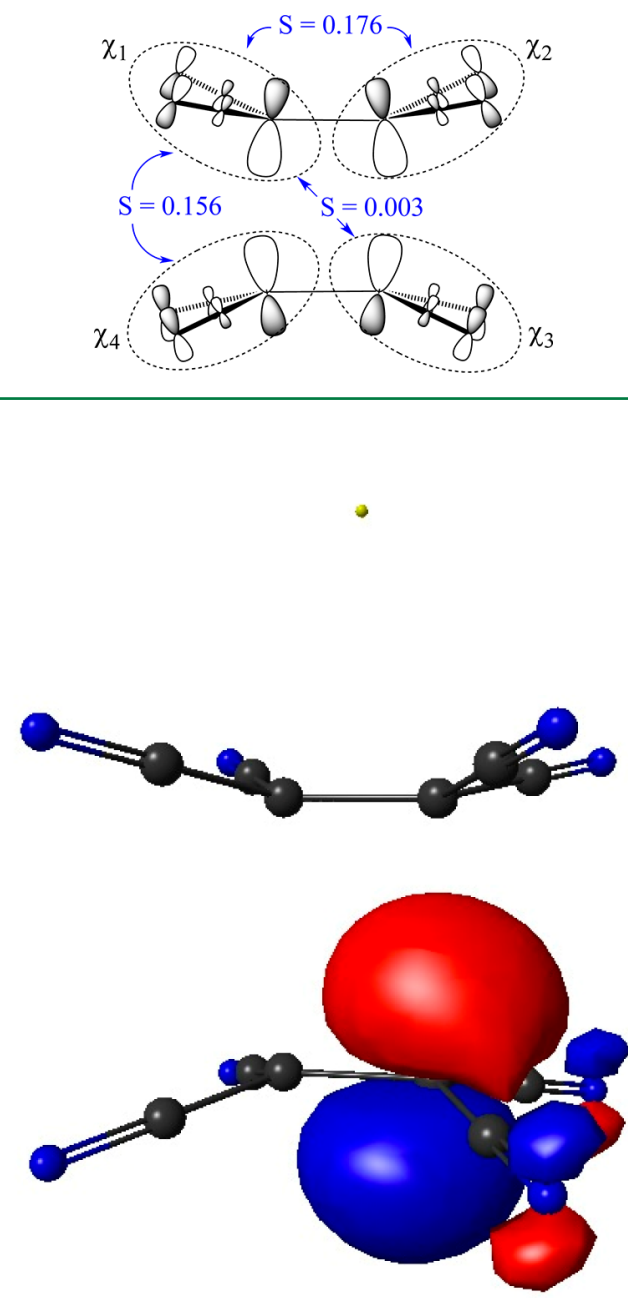

Figure 2. One of the four fragment orbitals involved in the $\mathrm{VB}$ calculations of $\mathrm{Na}_{2}{ }^{+}[\mathrm{TCNE}]_{2}{ }^{2-}$. The three other ones are identical on each $\mathrm{C}(\mathrm{CN})_{2}$ fragment.

one of them is shown in Figure 2. All these active orbitals are equivalent and deduced from each other by a symmetry operation. It can be seen in Figure 2 that each $\chi_{i}$ orbital is significantly delocalized over the $\mathrm{CN}$ substituents, i.e., moved away from the center of the $\mathrm{C}-\mathrm{C}$ link in each TCNE fragment, relative to an unsubstituted ethylenic unit. This has the consequence that the intrafragment overlaps $S_{12}$ and $S_{34}$ are quite weak, 0.176, and hardly larger than the interfragment overlaps $S_{14}$ and $S_{23}, 0.156$, as shown in Scheme 5. As will be shown shortly in the Discussion, both this quasi-equality between intra- and interfragment orbital overlaps and this value of $\sim 0.16-0.17$ are more than mere coincidences and turn out to be fundamental features of the $[\mathrm{TCNE}]_{2}{ }^{2-}$ complex. 
The weights of the VB structures, as calculated at the VBVMC level, are displayed in Table 2, entry 1 . As anticipated, the

Table 2. Weights of the VB Structures 1-6, in the $\mathrm{Na}_{2}{ }^{+}[\mathrm{TCNE}]_{2}{ }^{2-}$ Complex, and in an Unsubstituted $\mathrm{Na}_{2}{ }^{+}\left[\mathrm{C}_{2} \mathrm{H}_{4}\right]_{2}{ }^{2-}$ Diethylene Complex, As Calculated at the VB-VMC/DZ Level $^{a}$

$\begin{array}{ccccc}\text { VB structures } & \mathbf{1 , 2} & \mathbf{3 , 4} & \mathbf{5 , 6} & \mathbf{7 - 1 0} \\ \mathrm{Na}_{2}{ }^{+}[\mathrm{TCNE}]_{2}{ }^{2-} & 20.2 & 16.1 & 10.1 & 1.75 \\ \mathrm{Na}_{2}{ }^{+}[\mathrm{TCNE}]_{2}{ }^{2-b} & 21.7 & 17.3 & 10.9 & \text { n/a } \\ \mathrm{Na}_{2}{ }^{+}\left[\mathrm{C}_{2} \mathrm{H}_{4}\right]_{2}{ }^{2-} & 21.9 & 21.1 & 7.1 & \text { n/a }\end{array}$

${ }^{a_{\text {The }}}$ weights are given in $\%$ units and sum up to $100 \% .{ }^{b}$ Weights of 1-6 from entry 1 , renormalized to unity.

closed shell structures 7-10 are practically negligible, with a weight of $1.75 \%$ each. Accordingly, the analysis of the VB weights will focus on a simplified wave function, restricted to structures 1-6, entry 2 of Table 2 . It can be seen that structures $\mathbf{1}$ and $\mathbf{2}$ and structures $\mathbf{3}$ and $\mathbf{4}$ are clearly the major ones, with weights of $21.7 \%$ and $17.3 \%$, respectively. It is noteworthy that these two sets of VB structures have clearly different weights, at variance with Hartree-Fock or GVB wave functions as the latter appear once mapped on VB structures (vide supra). These unequal weights make sense physically, since the two fragment ionic charges borne by the $\pi$ lone pairs are facing each other in $\mathbf{3}$ and $\mathbf{4}$, which is destabilizing relative to $\mathbf{1}$ and $\mathbf{2}$ where the lone pairs are remote from each other (see Scheme 6). The

Scheme 6. Interacting Instantaneous Dipoles in Structures 1-4

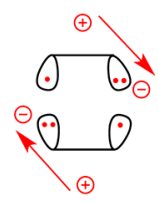

1

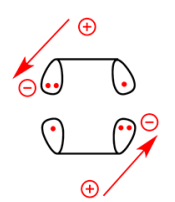

2

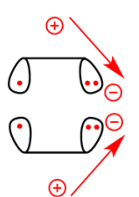

3

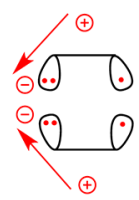

4 $\mathbf{1}$ and $\mathbf{2}$ vs 3 and $\mathbf{4}$ difference may also be understood in terms of induced-dipole-induced-dipole interactions. Indeed, the two TCNE fragments exhibit two dipoles in a stabilizing arrangement due to their opposite directions in $\mathbf{1}$ and $\mathbf{2}$, while $\mathbf{3}$ and $\mathbf{4}$ are destabilized with their two dipoles pointing in the same direction, as shown in Scheme 6. Thus, the unequal weights of $\mathbf{1}$ and 2 vs 3 and 4 reflect some instantaneous correlated fluctuations of two electrons, one on each monomer. Last, the charge transfer structures $\mathbf{5}$ and $\mathbf{6}$ also have quite significant weights, $10.9 \%$ each, an important feature that will be discussed in the next section.

An alternative way to appreciate the importance of a VB structure is to calculate the stabilization that results from its contribution to the ground state. By removing the structure from the wave function, and comparing the energy of the reduced wave function to that of the full multistructure, one can easily estimate this stabilization. As seen in Table 1 , the wave function reduced to structures $\mathbf{1 - 6}$ is only $1.7 \mathrm{kcal} / \mathrm{mol}$ higher than the full wave function involving structures 1-10, showing that $7-10$ are negligible indeed. On the other hand, removing 5 and 6 from 1-6 results in a significant destabilization, 8.9 $\mathrm{kcal} / \mathrm{mol}$, such that the resulting wave function involving only 1-4 is now repulsive. Therefore, the decisive stabilizing effects of $\mathbf{5}$ and $\mathbf{6}$, as well as their significant weights, is clear proof that these two VB structures, which now have nothing to do with dispersion, are essential ingredients of the electronic structure of the $\mathrm{Na}_{2}{ }^{+}[\mathrm{TCNE}]_{2}^{2-}$ complex.

\section{DISCUSSION}

As shown above, even with cations in an axial conformation, in which the electrostatic interaction is not stabilizing, the two anionic TCNE fragments are linked by a significant multicenter bonding interaction that is captured by a simple description in terms of the six VB structures $\mathbf{1 - 6}$. The information provided by this compact wave function can now be used to better understand the nature of the bond that holds together the two fragments.

A Mere Covalent Two-Electron Bond? As shown by several authors, a simple covalent two-electron bond between the $\pi^{*}$ orbitals of the TCNE units is unable to hold the two anionic fragments together in the $[\mathrm{TCNE}]_{2}{ }^{2-}$ dianion. Furthermore, the unusually large contribution of dynamic correlation to bonding was found to be incompatible with covalent CC bonding. ${ }^{7,10,11}$ Our VB calculations provide additional arguments to rule out the two-electron covalent bonding model. Besides the fact that a GVB wave function, which is normally very well suited for a two-electron covalent bond, would correspond to a VB function with all structures 14 having the same weights, at variance with our more accurate $\mathrm{VB}$ results, another intriguing fact is the large weight of structures $\mathbf{5}$ and $\mathbf{6}$. In a two-electron bonding scheme involving a covalent bond between the $\pi^{*}$ MOs of each monomer, 5 and 6 would be the charge-transfer components of the bond. However, such components are normally very small for standard CC bonds at large interatomic separations, as is the case here. Indeed, in an ethane molecule stretched to $2.56 \AA$ and distorted so as to mimic the geometry of $[\mathrm{TCNE}]_{2}{ }^{2-}$, the weight of charge-transfer structures (displaying ionic bonds $\mathrm{C}^{+} \mathrm{C}^{-}$and $\mathrm{C}^{-} \mathrm{C}^{+}$) is only $4.8 \%$ as calculated at the VBSCF level, less than half the weights of structures 5 and $\mathbf{6}$. Clearly then, the large weights of these charge-transfer structures are not compatible with a mere two-electron bond and must be the signature of an alternative bonding scheme.

A Combination of Three-Electron Bonds? Threeelectron bonding is common in chemistry and biochemistry. Examples include many radical cations or anions, ${ }^{35,36}$ among which are the disulfide $S .: S$ bridge in reduced biochemical compounds, and the $\pi$ bonds of dioxygen. Before examining the three-electron bonding hypothesis in the case at hand, we briefly recall the main features of this type of bonding. Let us consider two atoms or fragments $\mathrm{A}^{\bullet}$ and $\mathrm{A}^{\prime \bullet}$, each displaying a singly occupied orbital. Adding one electron to this system yields an anion, in which $\mathrm{A}$ and $\mathrm{A}^{\prime}$ can be linked by a threeelectron bond, noted $\left[\mathrm{A} \cdot \mathrm{A}^{\prime}\right]^{-}$, as in eq 5 :

$$
\Psi\left(\mathrm{A} \therefore \mathrm{A}^{\prime}\right)^{-}=\mathrm{A}^{-{ }^{-}} \mathrm{A}^{\prime} \leftrightarrow \mathrm{A}^{\bullet}: \mathrm{A}^{\prime-}
$$

Here, the wave function is made of two interacting Lewis structures. In $\left(\mathrm{A}^{-{ }^{-}} \mathrm{A}^{\prime}\right)$, A displays a doubly occupied orbital facing the singly occupied orbital of $\mathrm{A}^{\prime}$, and the reverse is true in $\left(\mathrm{A}^{\bullet}: \mathrm{A}^{\prime-}\right)$.

The two Lewis structures corresponding to VB description of a $\pi$ three-electron bond in the ethylene anion are depicted in Scheme 3a. The corresponding molecular orbital picture is shown in Scheme 3b. It is important to note that each of these two Lewis structures, taken alone, is unbound. Indeed, in elementary VB theory, ${ }^{19}$ it is known that such a system displays a three-electron repulsion of $-2 \beta S /\left(1-S^{2}\right)(\beta$ is a negative 
reduced resonance integral as in Hückel theory, and $S$ is the overlap between the interacting atomic orbitals). Therefore, the bonding energy is entirely due to resonance between the two Lewis structure, leading to a bonding energy $\beta(1-3 S) /(1-$ $\left.S^{2}\right)$ relative to the separate fragments. ${ }^{19}$ It has been shown ${ }^{37}$ that the bonding energy is greatest when the orbital overlap $S \approx$ $1 / 6$, a rather small value which explains why 3-e $\sigma$ bonds are always long bonds.

Turning now to the TCNE monomers, it is clear that each of them displays an intrafragment 3-e bond in its $\pi$ system, as in Scheme $3 a$, when they are at infinite distance from each other. In terms of VB structures for the entire dimer, the interacting combination $\mathbf{2} \leftrightarrow \mathbf{3}$ (or $\mathbf{1} \leftrightarrow \mathbf{4}$ ) describes an internal 3-e $\pi$ bond between orbitals $\chi_{1}$ and $\chi_{2}$ in the upper fragment, while the orbital occupancies of $\chi_{3}$ and $\chi_{4}$ in the lower fragment are frozen. Conversely, $\mathbf{1} \leftrightarrow \mathbf{3}$ (or $\mathbf{2} \leftrightarrow \mathbf{4}$ ) describes an internal 3-e $\pi$ bond in the lower fragment. Thus, the supersystem with infinite interfragment separation displays a pair of intrafragment 3-e bonds that is well described by structures $1-4$ with equal weights. Then, as the interfragment distance is reduced, the $\left\langle\chi_{1} \mid \chi_{4}\right\rangle$ and $\left\langle\chi_{2} \mid \chi_{3}\right\rangle$ interfragment overlaps increase while the cross overlaps stay close to zero (see Scheme 5). Finally, at equilibrium distance, the inter- and intrafragment orbital overlaps become nearly equivalent, respectively 0.156 and 0.176 , implying that the $\chi_{1}-\chi_{4}$ and $\chi_{2}-\chi_{3}$ 3-e interactions become comparable to the $\chi_{1}-\chi_{2}$ and $\chi_{3}-\chi_{4}$ ones. It follows that the intrafragment 3 -e $\pi$ bonds that were unique at infinite separation now coexist with interfragment 3-e $\sigma$ bonds, as represented in Scheme 7. Remarkably, the equilibrium structure

Scheme 7. Representation of the $[\mathrm{TCNE}]_{2}{ }^{2-}$ Complex in Terms of Interacting Intrafragment Three-Electron $\pi$ Bonds (a) and Interfragment Three-Electron $\sigma$ Bonds (b)

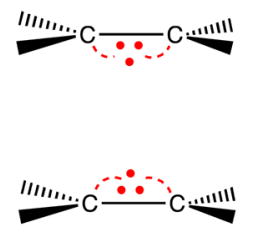

(a)

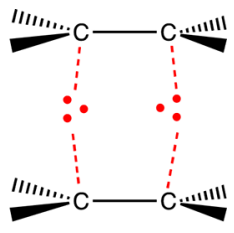

(b) is reached when the interfragment orbital overlaps, $\left\langle\chi_{1} \mid \chi_{4}\right\rangle$ and $\left\langle\chi_{2} \mid \chi_{3}\right\rangle$, are almost equal to the "magic" value $1 / 6$ that ensures maximum stabilization for a 3-e interaction.

In the same way as the intrafragment 3-e $\pi$ bonds (Scheme 7a) are represented by $1-4$, the interfragment 3-e $\sigma$ bonds (Scheme $7 b$ ), which hold the two fragments together, are represented by $1,2,5$, and 6 . Thus, the dianion complex is the site of a competition between intrafragment and interfragment 3-e bonds. Because 5 and $\mathbf{6}$ are unique to the interfragment 3-e bonds while 3 and 4 are unique to the intrafragment ones, the ratio of $5+6$ vs $3+4$ weights can measure the ratio of inter- vs intrafragment bonds. As a result, the ratio of interfragment to intrafragment bonding amounts to $38.5 \%$ vs $61.5 \%$ (normalized to unity) in the $[\mathrm{TCNE}]_{2}^{2-}$ system at equilibrium, at the VB$\mathrm{VMC} / \mathrm{DZ}$ level. This bonding scheme immediately explains the large weights of the charge transfer structures 5 and $\mathbf{6}$ and is also consistent with the weights of $\mathbf{1}$ and $\mathbf{2}$ being larger than those of 3 and 4.

The description of the above dianionic complexes in terms of six main VB structures and the simple 3-e bonding model can now be compared with various features of these unusual compounds. The first feature relates to their intriguing interfragment bond length, which is too short for a van der Waals interaction but too long for covalent $\mathrm{C}-\mathrm{C}$ bonding. As noted above, such bond lengths fall within the range expected for 3-e $\mathrm{C}-\mathrm{C}$ bonds, in the same way as the interfragment orbital overlaps are typical of this type of bonding. A second aspect of the $[\mathrm{TCNE}]_{2}{ }^{2-}$ dianion is the overwhelming importance of dynamic electron correlation, estimated to contribute $23 \mathrm{kcal} / \mathrm{mol}$ to bonding. ${ }^{7}$ Once again, this is in perfect agreement with the 3-e bonding model. Indeed, all the electron correlation of the active electrons in such bonds is of a dynamic nature and has been shown to contribute some 16-28 $\mathrm{kcal} / \mathrm{mol}$ to bonding in 3-e homonuclear bonds of first rowcompounds (from $\mathrm{N}$ to $\mathrm{Ne}$ ). ${ }^{18}$

An Aromatic System. Another aspect of bonding in the $[\mathrm{TCNE}]_{2}^{2-}$ system becomes apparent when one considers the overlaps between the active orbitals at equilibrium distance, Scheme 5. As has been noted, the overlaps between neighboring orbitals are nearly the same, $0.166 \pm 0.01$, both almost identical to the "magic" theoretical value of $1 / 6$ where a three-electron bonding interaction is maximal, ${ }^{37}$ while the cross overlaps (e.g., $\left.\left\langle\chi_{1} \mid \chi_{3}\right\rangle\right)$ are negligible. Particularly striking is the fact that, in terms of overlaps, this makes the four-orbital/6-e system topologically equivalent to the $n$ system of the $\mathrm{C}_{4} \mathrm{H}_{4}{ }^{2-}$ aromatic ring, which is stable when complexed by two cations, one above and one below the ring, in perfect analogy with the $\mathrm{Na}_{2}{ }^{+}[\mathrm{TCNE}]_{2}{ }^{2-}$ system! This dianion has been studied by Jung et al. $^{38}$ and was shown to possess all the characteristics of an aromatic system: equal bond lengths, a negative Nuclear Independent Chemical Shift (NICS), and significant aromatic stabilization energy. It is therefore quite conceivable that "pancake bonding" is reinforced by aromaticity in the TCNE anion dimer, whose active electronic system complies to Hückel's “ $4 n+2$ " rule.

What Is the Role of the CN Substituents? To elucidate the role of the $\mathrm{CN}$ substituents in the $\mathrm{Na}_{2}{ }^{+}[\mathrm{TCNE}]_{2}{ }^{2-}$ complex, we performed a VB study of a simpler model system, $\mathrm{Na}_{2}{ }^{+}\left[\mathrm{C}_{2} \mathrm{H}_{4}\right]_{2}^{2-}$, made of two unsubstituted planar ethylene anions with $\mathrm{Na}^{+}$countercations in axial positions (Scheme 8).

Scheme 8. The Unsubstituted $\mathrm{Na}_{2}{ }^{+}\left[\mathrm{C}_{2} \mathrm{H}_{4}\right]_{2}{ }^{2-}$ Complex, with the Overlaps between the $\pi$ Atomic Orbitals

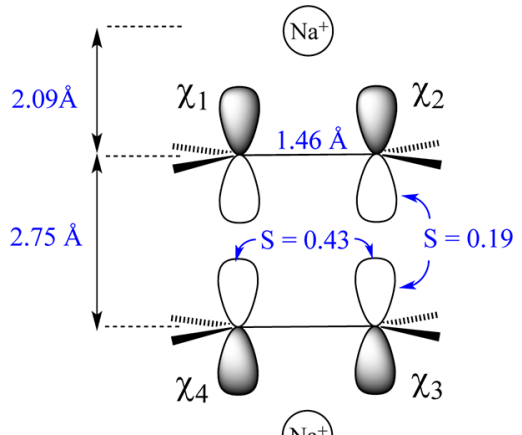

$\mathrm{Na}^{+}$

A set of geometries for the $\mathrm{Na}_{2}{ }^{+}\left[\mathrm{C}_{2} \mathrm{H}_{4}\right]_{2}{ }^{2-}$ complex were optimized in the standard $6-31 \mathrm{G}^{*}$ polarized double- $\zeta$ basis set at the MP2 level of theory for a series of fixed interfragment distances ranging from 2.3 to $4.7 \AA^{39}$ Then, using these MP2 geometries the dissociation curve was calculated at the CP$\operatorname{CCSD}(\mathrm{T})$ level using a cc-pVTZ basis set and counterpoise 
corrections for the BSSE. The CP-CCSD(T) curve, which is displayed in the Supporting Information (SI) document, reveals a geometrical minimum for an interfragment distance of $2.75 \AA$. However, this minimum is only a secondary one, as the dissociation of this model complex is exothermic. At this computational level, the $\mathrm{Na}_{2}{ }^{+}\left[\mathrm{C}_{2} \mathrm{H}_{4}\right]_{2}{ }^{2-}$ model complex is metastable, lying $10.9 \mathrm{kcal} / \mathrm{mol}$ above the separate fragments, with a barrier to dissociation of $4.7 \mathrm{kcal} / \mathrm{mol}$, that is reached at an interfragment distance of $3.85 \AA$. Such a secondary minimum is typical of bonding interactions between ions and results from the superposition of an attractive bonding interaction over a purely repulsive electrostatic curve. This indicates that there is indeed a bonding interaction between the two $\mathrm{C}_{2} \mathrm{H}_{4}{ }^{-\bullet}$ units, but too weak to overcome the repulsive electrostatic interaction in the axial conformation of the $\mathrm{Na}^{+}$ cations.

Table 2 also displays the weights of VB structures 1-6 for the diethylene system (entry 3), as calculated at the VB-VMC level with the same double- $\zeta$ as used in the real $\mathrm{Na}_{2}{ }^{+}[\mathrm{TCNE}]_{2}{ }^{2-}$ system. By comparison with the real system, the diethylene system exhibits two differences: (i) the weights of structures $\mathbf{5}$ and $\mathbf{6}$ are smaller (only 7\% each) and (ii) the weights of structures 1 and $\mathbf{2}$ and structures 3 and 4 are almost the same. These two features reduce the interfragment 3-e bonding component in the diethylene system relative to the $\mathrm{Na}_{2}{ }^{+}[\mathrm{TCNE}]_{2}^{2-}$ and consequently reduce the strength of the bonding interaction in the former. The reason for this poor bonding interaction becomes clear when one considers the orbital overlaps between the active AOs in the diethylene system, Scheme 8 . It can be seen that the intrafragment overlaps, $\left\langle\chi_{1} \mid \chi_{2}\right\rangle$ and $\left\langle\chi_{3} \mid \chi_{4}\right\rangle$, are now much stronger than the interfragment ones, 0.43 vs 0.19 . Such a strong overlap between two adjacent $\pi$ AOs of the same monomer necessarily disfavors structures 5 and 6 , in which the two negative charges are concentrated in the same $\mathrm{C}_{2} \mathrm{H}_{4}$ unit. As a consequence, poor bonding between the two ethylene anions results since, as we have seen, structures $\mathbf{5}$ and $\mathbf{6}$ are essential for 3-e interfragment bonding. This brings some new light onto the effect of the $\mathrm{CN}$ substituents to strengthen the interfragment bond. Of course, these substituents may strengthen this bond by making the $\beta$ Hückel parameter of carbon more negative, as it is well-known that 3 -e bonding is stronger between more electronegative atoms. ${ }^{35,36}$ However, another facet of the role of $\mathrm{CN}$ substituents consists of delocalizing the active fragment orbitals so as to put them apart from each other and lower their mutual overlap. This effect stabilizes structures $\mathbf{5}$ and $\mathbf{6}$, which can now come into play and contribute to 3 -e bonding between the two fragments.

An alternative way to view the difference between this model and the real system calls for the analogy with anionic $\mathrm{C}_{4} \mathrm{H}_{4}$ rings. As has been said, the $\mathrm{Na}_{2}{ }^{+}[\mathrm{TCNE}]_{2}{ }^{2-}$ system with its quasi-identical overlaps between neighboring orbitals is topologically equivalent to a square $\mathrm{C}_{4} \mathrm{H}_{4}{ }^{2-}$ aromatic ring. On the other hand, the diethylene system with its alternated overlaps (Scheme 8) is rather analogous to a $\mathrm{C}_{4} \mathrm{H}_{4}{ }^{2-}$ ring that would have been strongly deformed to a rectangular structure, thus losing its aromatic properties. From this topological analogy, the role of the $\mathrm{CN}$ substituents could be viewed as delocalizing the fragment orbitals up to the point where the intra- and interfragment orbital overlaps become equal, thus endowing the $\mathrm{Na}_{2}{ }^{+}[\mathrm{TCNE}]_{2}{ }^{2-}$ complex with aromatic properties.

\section{CONCLUSION}

The ditetracyanoethylene dianion complexed by a pair of sodium cations has been calculated at the $\operatorname{CCSD}(\mathrm{T})$ level and at $a b$ initio VB levels including both static and dynamic electron correlation. In agreement with previous calculations at the MCQDPT/CASSCF (2,2) level, ${ }^{7}$ the $\operatorname{CCSD}(\mathrm{T})$ reference calculations show that a bonding interaction exists between the two TCNE anions, as shown by the existence of a true minimum on the dissociation curve, lying ca. $10 \mathrm{kcal} / \mathrm{mol}$ lower than the dissociation products, despite the axial geometrical conformation that has been chosen in order to eliminate the electrostatic contribution to bonding. The VB calculations show that the essential physics of the multicenter bonding is captured by a very simple description in terms of only six VB structures, provided electron correlation of all electrons, and most importantly dynamic electron correlation, is included in the compact wave function, as is done when VB is coupled with QMC theory. The VB picture is consistent with a dispersionassisted bonding interaction between the two TCNE anions, in agreement with the conclusions of other authors.,10,11 However, the bonding interaction that takes place between the $\pi$ systems of the two anions cannot be of the two-electron covalent type, as indicated by the large weights of the chargetransfer VB structures that sum up to $21.7 \%$ and are not compatible with a stretched covalent bond at this long interatomic distance. On the other hand, the weights of the VB structures are consistent with a simple picture in terms of two three-electron-bonded cartoons, one displaying a pair of interfragment 3-e $\sigma$ bonds and the other displaying intrafragment 3-e $\pi$ bonds, as illustrated in Scheme 7. This simple picture explains at once (1) the long interfragment bond length, which is independent of the counter-cations but typical of 3-e CC $\sigma$ bonds, (2) the interfragment orbital overlaps which are very close to the theoretical optimal overlap of $1 / 6$ for a 3-e $\sigma$ bond, and (3) the unusual importance of dynamic correlation, which is precisely the main bonding component of 3-e bonds.

Interestingly, the intrafragment orbital overlaps are also very close to the same value of $1 / 6$, while the cross overlaps are negligible. This makes the $\mathrm{Na}_{2}{ }^{+}[\mathrm{TCNE}]_{2}{ }^{2-}$ system topologically equivalent to the square $\mathrm{C}_{4} \mathrm{H}_{4}{ }^{2-}$ dianion, surrounded by two cations in the axial position. Indeed, both systems have six active electrons, four active orbitals, and equal overlaps between neighboring orbitals. As the $\mathrm{Li}_{2}{ }^{+} \mathrm{C}_{4} \mathrm{H}_{4}{ }^{2-}$ complex has been shown to have all the characteristics of an aromatic system, in compliance to the " $4 n+2$ " Hückel rule, it is clear that aromaticity must also play a significant role in the remarkable stability of the $\mathrm{Na}_{2}{ }^{+}[\mathrm{TCNE}]_{2}{ }^{2-}$ complex. In this respect, it is interesting to note a formal analogy between the active electronic system of [TCNE $]_{2}^{2-}$ and the $\pi$-electronic system of $\mathrm{S}_{2} \mathrm{~N}_{2}$ and related systems $\left(\mathrm{S}_{2} \mathrm{E}_{2}\right.$ and $\left.\mathrm{E}_{4}{ }^{2+}, \mathrm{E}=\mathrm{S}, \mathrm{Se}, \mathrm{Te}\right)$, which also involve six electrons over four centers. ${ }^{40}$ These latter molecules have also been shown to possess significant aromaticity, based on both magnetic ${ }^{41}$ and energetic criteria. ${ }^{40}$

The analogy between $\mathrm{C}_{4} \mathrm{H}_{4}{ }^{2-}$ and $[\mathrm{TCNE}]_{2}^{2-}$ also makes clear the role of the cyano substituents and why a naked diethylene dianion complex, even surrounded by two axial cations, is only metastable. Such a complex exhibits very different overlaps between neighboring active orbitals, according as the orbitals belong to the same ethylenic fragment or face each other along an axial direction. This makes the system analogous to a rectangular $\mathrm{C}_{4} \mathrm{H}_{4}{ }^{2-}$ dianion, therefore devoid of significant aromaticity. The role of the cyano $\pi$-attractive 
substituents is therefore to move the fragment orbitals away from each other inside each ethylenic fragment, thus lowering their mutual overlap so that all orbital overlaps can be equalized, a necessary condition for aromaticity to be effective. These mutual overlaps are necessarily small and close to $1 / 6$, as required for interfragment 3-e $\sigma$ bonding to be strongest. To achieve this overlap equalization, the ethylenic units must be substituted with very strong $\pi$ attractors, a condition that is met in each tetracyanoethylene fragment.

It is clear from the above picture that all six active electrons contribute to interfragment bonding. Consequently, the "2-e/4c" label for the $[\mathrm{TCNE}]_{2}{ }^{2-}$ bond would better be replaced by "6-e/4-c". More generally, the " $n$-e/m-c" label for related systems might also be deemed inappropriate. In line with this simple picture, we conclude that the so-called 2-e/4-c bonding in the $[\mathrm{TCNE}]_{2}{ }^{2-}$ dianion is not to be considered as a new type of bond, since the fragments are held together by a resonating combination of classical 3-e bonds, a type of bond that was described for the first time by Pauling in $1931 .^{42}$ Dispersion forces obviously also play a role, even if we cannot clearly separate these forces from other bonding interactions. Incidentally, because 3-e bonds can be considered as a particular case of charge-shift bond (bonds in which the resonance energy is the major cause for bonding), ${ }^{16}$ our results formally agree with the suggestion of Tian and Kertesz that charge-shift bonding contributes to the chemical bonding in $[\mathrm{TCNE}]_{2}{ }^{2-}$ dimers. $^{17}$

\section{ASSOCIATED CONTENT}

\section{Supporting Information}

Detailed calculations and dissociation curves for the model system $\mathrm{Na}_{2}{ }^{+}\left[\mathrm{C}_{2} \mathrm{H}_{4}\right]_{2}^{2-}$.

\section{AUTHOR INFORMATION}

\section{Corresponding Author}

*Fax: +33 (0)169154447. E-mail: benoit.braida@upmc.fr, philippe.hiberty@u-psud.fr.

\section{Present Address}

"Laboratoire de Modélisation et Simulation Multi Echelle, Equipe de Chimie Théorique, Université Paris-Est Marne-laVallée, 5, Boulevard Descartes, 77454 Marne-la-Vallée Cedex 2, France

\section{Notes}

The authors declare no competing financial interest.

\section{ACKNOWLEDGMENTS}

Prof. Wei Wu and Cyrus J. Umrigar are gratefully acknowledged for making their XMVB and CHAMP respective codes available to us. Computing facilities by IDRIS are also acknowledged.

\section{REFERENCES}

(1) Lemervovskii, D. A.; Stukan, R. A.; Tarasevich, B. N.; Slovokhotov, Tu. L.; Antipin, M. Yu.; Kalinin, A. E.; Struchov, Yu. T. Struk. Chim. 1981, 7, 240-249.

(2) (a) Gundel, D.; Sixl, H.; Metzger, R. M.; Heimer, N. E.; Harms, R. H.; Keller, H. J.; Nothe, D.; Wehe, D. J. Chem. Phys. 1983, 79, 3678. (b) Miller, J. S.; Zhang, J. H.; Reiff, W. M.; Dixon, D. A.; Preston, L. D.; Reis, A. H., Jr.; Gebert, E.; Extine, M.; Troup, J.; Epstein, A.; Ward, M. D. J. Phys. Chem. 1987, 91, 4344. (c) Chesnut, D. B.; Phillips, W. D. J. Chem. Phys. 1961, 35, 1002.
(3) (a) Miller, J. S.; Krusic, P. J.; Dixon, D. A.; Reiff, W. M.; Zhang, J. H.; Anderson, E. C.; Epstein, A. J. J. Am. Chem. Soc. 1986, 108, 4459.

(b) Pasimeni, L.; Brustolon, M.; Zanonato, P. L.; Corvaja, C. Chem. Phys. 1980, 51, 381.

(4) Lu, J.-M.; Rosokha, S. V.; Kochi, J. K. J. Am. Chem. Soc. 2003, 125,12161 and references therein.

(5) Novoa, J. J.; Lafuente, P.; Del Sesto, R. E.; Miller, J. S. Angew. Chem., Int. Ed. 2001, 40, 2540-2544.

(6) Del Sesto, R. E.; Miller, J. S.; Lafuente, P.; Novoa, J. J. Chem.Eur. J. 2002, 8, 21.

(7) Jung, Y.; Head-Gordon, M. Phys. Chem. Chem. Phys. 2004, 6, 2008-2011.

(8) Garcia-Yoldi, I.; Miller, J. S.; Novoa, J. J. J. Phys. Chem. A 2007, $111,8020-8027$.

(9) Novoa, J. J.; Ribas-Arino, J.; Shum, W. W.; Miller, J. S. Inorg. Chem. 2007, 46, 103-107.

(10) Mota, F.; Miller, J. S.; Novoa, J. J. J. Am. Chem. Soc. 2009, 131, 7699-7707.

(11) Garcia-Yoldi, I.; Mota, F.; Novoa, J. J. J. Comput. Chem. 2007, $28,326-334$.

(12) Miller, J. S.; Novoa, J. J. Acc. Chem. Res. 2007, 40, 189-196.

(13) Del Sesto, R. E.; Miller, J. S.; Novoa, J. J.; Lafuente, P. Chem.Eur. J. 2002, 8, 4894-4908.

(14) Bader, R. F. W. Atoms in Molecules, X-Ray Analyses and the Structure of Organic Molecules; Clarendon Press: Oxford, U. K., 1990; $\mathrm{p}$ 32.

(15) Novoa, J. J.; Stephens, P. W.; Weerasekare, M.; Shum, W. W.; Miller, J. S. J. Am. Chem. Soc. 2009, 131, 9070-9075.

(16) (a) Shaik, S.; Danovich, D.; Silvi, B.; Lauvergnat, D.; Hiberty, P. C. Chem.-Eur. J. 2005, 11, 6358. (b) Shaik, S.; Danovich, D.; Wu, W.; Hiberty, P. C. Nat. Chem. 2011, 1, 443.

(17) Tian, Y.-H.; Kertesz, M. J. Chem. Phys. A 2011, 115, 1394213949.

(18) (a) Hiberty, P. C.; Humbel, S.; Archirel, P. J. Phys. Chem. 1994, 98, 11697. (b) Hiberty, P. C.; Humbel, S.; Danovich, D.; Shaik, S. J. Am. Chem. Soc. 1995, 117, 9003.

(19) Shaik, S.; Hiberty, P. C. A Chemist's Guide to Valence Bond Theory; Wiley-Interscience: New York, 2007.

(20) Shaik, S.; Hiberty, P. C. Rev. Comput. Chem. 2004, 20, 1-100.

(21) Wu, W.; Mo, Y.; Cao, Z.; Zhang, Q. In Valence Bond Theory; Cooper, D. L., Ed.; Elsevier: Amsterdam, The Netherlands, 2002; pp 143-185.

(22) Chirgwin, H. B.; Coulson, C. A. Proc. R. Soc. London, Ser. A 1950, 201, 196-209.

(23) Hiberty, P. C.; Shaik, S. J. Comput. Chem. 2007, 137, 28.

(24) (a) van Lenthe, J. H.; Balint-Kurti, G. G. Chem. Phys. Lett. 1980, 76, 138-142. (b) van Lenthe, J. H.; Balint-Kurti, G. G. J. Chem. Phys. 1983, 78, 5699-5713. (c) Verbeek, J.; van Lenthe, J. H. J. Mol. Struct. (THEOCHEM) 1991, 75, 115-137.

(25) Braida, B.; Toulouse, J.; Caffarel, M.; Umrigar, C. J. J. Chem. Phys. 2011, 134, 084108.

(26) Toulouse, J.; Umrigar, C. J. J. Chem. Phys. 2008, 128, 174101.

(27) Burkatzki, M.; Filippi, C.; Dolg, M. J. Chem. Phys. 2007, 126, 234105 .

(28) Basis sets and corresponding pseudopotentials are available online: http://burkatzki.com/pseudos/index.2.html, 2008.

(29) Song, L.; Mo, Y.; Zhang, Q.; Wu, W. XMVB-An Ab initio Nonorthogonal Valence Bond; Xiamen University: Xiamen, China, 2003.

(30) (a) Song, L.; Mo, Y.; Zhang, Q.; Wu, W. J. Comput. Chem. 2005, 26, 514-521. (b) Song, L.; Song, J.; Mo, Y.; Wu, W. J. Comput. Chem. 2009, 30, 399-406.

(31) Umrigar, C. J., Filippi, C.; Toulouse, J. CHAMP, a Quantum Monte Carlo ab initio program, 2012. http://pages.physics.cornell. edu/ cyrus/champ.html.

(32) Lee, T. J.; Rice, J. E.; Scuseria, G. E.; Schaefer, H. F. Theor. Chim. Acta 1989, 75, 81.

(33) Lee, T. J.; Taylor, P. R. Int. J. Quantum Chem. 1989, 23, 199.

(34) A detailed method for mapping MO configurations to VB wave functions is described in chapter 4 of ref 19. In the present case, the 
method can be greatly simplified by using the hole-particle equivalence. By exchanging electrons with holes in the four-orbital six-electron system, one deals with simple four-orbital two-electron determinants with which the mapping procedure is immediately done by hand. Then, one only has to do the back hole-particle transformation to get the results in terms of four-orbital six-electron determinants.

(35) Gill, P. M. W.; Radom, L. J. Am. Chem. Soc. 1989, 111, 4613.

(36) (a) Clark, T. J. Am. Chem. Soc. 1988, 110, 1672. (b) Gill, P. M. W.; Radom, L. J. Am. Chem. Soc. 1988, 110, 4931.

(37) Baird, N. C. J. Chem. Educ. 1977, 54, 291.

(38) Jung, Y.; Heine, T.; Schleyer, P. v. R.; Head-Gordon, M. J. Am. Chem. Soc. 2004, 126, 3132.

(39) Only the core basis functions for $\mathrm{Na}^{+}$have been used in the calculations, in order to avoid significant charge transfer between the $\mathrm{Na}^{+}$cations and the ethylene anion fragments.

(40) Braida, B.; Lo, A.; Hiberty, P. C. ChemPhysChem 2012, 13, 811-819.

(41) Jung, Y.; Heine, T.; Schleyer, P. v. R.; Head-Gordon, M. J. Am. Chem. Soc. 2004, 126, 3132.

(42) (a) Pauling, L. J. Am. Chem. Soc. 1931, 53, 3225. (b) Pauling, L. J. Chem. Phys. 1933, 1, 56. 